EPJ Web of Conferences 73, 05007 (2014)

DOI: $10.1051 /$ epjconf/20147305007

(C) Owned by the authors, published by EDP Sciences, 2014

\title{
Interplay of antikaons with hyperons in nuclei and in neutron stars
}

\author{
Takumi Muto ${ }^{1, a}$, Toshiki Maruyama ${ }^{2}$ and Toshitaka Tatsumi ${ }^{3}$ \\ ${ }^{1}$ Physics Division, Education Center, Chiba Institute of Technology, 2-1-1 Shibazono, Narashino, \\ Chiba 275-0023, Japan \\ ${ }^{2}$ Advanced Science Research Center, Japan Atomic Energy Agency, Ibaraki 319-1195, Japan \\ ${ }^{3}$ Department of Physics, Kyoto University, Kyoto 606-8502, Japan
}

\begin{abstract}
Possible coexistence of antikaons and hyperons in nuclei and in neutron stars is investigated based on the same interaction model within the relativistic mean-field theory. It is shown that the ground state of multi-strangeness nuclei is given by multi-hypernuclei without bound $K^{-}$mesons. In neutron stars, on the other hand, kaon condensation appears in hyperon-mixed matter for a moderate $K^{-}$potential depth, but it leads to significant softening of the equation of state. Some repulsive effect at high density should be taken into account so as to be consistent with recent observations of massive neutron stars.
\end{abstract}

\section{Introduction}

It has long been suggested that meson-baryon system with multi-strangeness should play an important role in exploring new aspect of hadron physics. For high-density matter in neutron stars, kaon-condensed phase in hyperon-mixed matter (abbreviated to $Y+K$ phase) has been considered, and its implications for astrophysical phenomena such as structure and thermal evolution of neutron stars have been discussed [1]. Recently we have studied multi-antikaonic nuclei (MKN), where several $K^{-}$mesons are bound in the nucleus, based on a relativistic mean-field (RMF) model for baryon-baryon $(B-B)$ interaction, combined with the effective chiral Lagrangian for kaon-baryon $(\bar{K}-B)$ and nonlinear $\bar{K}$ $\bar{K}$ interactions [2]. (Similar results have been obtained for the MKN in [3], where kaonic part of the Lagrangian corresponds to that obtained by the lowest-order expansion with respect to the kaon field.)

In this paper, $K^{-}$mesons and hyperons are taken into account together in a unified way for both nuclei and neutron stars based on the above model interaction: We study possible coexistence of $K^{-}$ mesons and hyperons in nuclei as the ground state of multi-strangeness nuclei [4]. We also obtain onset density and the equation of state (EOS) of the $Y+K$ phase in neutron stars, and compare kaon properties in such highly dense matter with those in finite nuclei.

\footnotetext{
ae-mail: takumi.muto@it-chiba.ac.jp
}

This is an Open Access article distributed under the terms of the Creative Commons Attribution License 4.0, which permits unrestricted use, distribution, and reproduction in any medium, provided the original work is properly cited. 


\section{Relativistic mean-field model combined with effective chiral Lagrangian}

In the RMF, $B-B$ interactions are mediated by exchange of the $\sigma, \sigma^{*}, \omega, \rho$, and $\phi$ mesons. $\bar{K}-B$ and $\bar{K}-\bar{K}$ interactions are introduced from the nonlinear effective chiral Lagrangian [5]. In our model, the original $s$-wave scalar and vector contact interactions between the nonlinear $\bar{K}$ field and baryons are replaced by those generated by the scalar $\sigma, \sigma^{*}$ mesons-exchange and vector $\omega, \rho$, and $\phi$ mesons-exchange, respectively. The $K^{-}$mesons are assumed to be condensed in the lowest-energy state. The $K^{-}$field is then given classically as $K(r) \equiv\left\langle K^{-}\left|\hat{K}^{-}\right| K^{-}\right\rangle=f \theta(r) / \sqrt{2}$, where $\theta(r)$ is the chiral angle and $f(=$ $93 \mathrm{MeV}$ ) the meson decay constant. After this prescription, the kaonic part of the Lagrangian density is represented as

$$
\begin{aligned}
\mathcal{L}_{K M}= & \frac{1}{2}\left\{1+\left(\frac{\sin \theta}{\theta}\right)^{2}\right\}\left(\mathcal{D}^{\mu} K\right)^{\dagger} \mathcal{D}_{\mu} K+\frac{1-\left(\frac{\sin \theta}{\theta}\right)^{2}}{2 f^{2} \theta^{2}}\left\{\left(K^{\dagger} \mathcal{D}_{\mu} K\right)^{2}+\left(K\left(\mathcal{D}_{\mu} K\right)^{\dagger}\right)^{2}\right\} \\
& -m_{K}^{* 2}\left(\frac{\sin (\theta / 2)}{\theta / 2}\right)^{2} K^{\dagger} K+i X_{0}\left(\frac{\sin (\theta / 2)}{\theta / 2}\right)^{2}\left(K^{\dagger} \mathcal{D}_{\mu} K-\left(\mathcal{D}_{\mu} K\right)^{\dagger} K\right),
\end{aligned}
$$

where $m_{K}^{* 2}\left[\equiv m_{K}^{2}-2 m_{K}\left(g_{\sigma K} \sigma+g_{\sigma^{*} K} \sigma^{*}\right)\right]$ is the square of the effective mass of the $K^{-}$, reflecting the decrease in the kaon mass due to the $K^{-}-B$ scalar interaction, and $X_{0} \equiv g_{\omega K} \omega_{0}+g_{\rho K} R_{0}+g_{\phi K} \phi_{0}$, standing for the $K^{-}-B$ vector interaction. The shift of $K^{-}$energy by the Coulomb field $A^{0}$ is introduced through the covariant derivative $\mathcal{D}^{\mu}=\partial^{\mu}-i e A^{\mu}$. In the expression of $m_{K}^{* 2}$ and $X_{0}, g_{M K}$ $\left(M=\sigma, \sigma^{*}, \omega, \rho, \phi\right)$ are the meson- $K^{-}$coupling constants, and $\sigma(r), \sigma^{*}(r), \omega_{0}(r), R_{0}(r)$, and $\phi_{0}(r)$ are the mean fields of the scalar mesons and time components of the vector mesons.

The values of the coupling constants for nucleon $(N)-N$ interaction, $g_{\sigma N}, g_{\omega N}, g_{\rho N}$, are determined so as to reproduce not only the properties of normal nuclear matter with saturation density $\rho_{0}=$ $0.153 \mathrm{fm}^{-3}$, but also the binding energy, proton-mixing ratio, and density distributions of proton and neutron for normal nuclei [2]. For the scalar meson couplings for hyperons $(Y)$, the potential depths of the $\Lambda, \Sigma^{-}$, and $\Xi^{-}$in symmetric nuclear matter are taken to be $U_{\Lambda}^{N}\left(\rho_{0}\right)=-27 \mathrm{MeV}$, $U_{\Sigma^{-}}^{N}\left(\rho_{0}\right)=23.5 \mathrm{MeV}$, and $U_{\Xi^{-}}^{N}\left(\rho_{0}\right)=-16 \mathrm{MeV}$, following the phenomenological analyses from the recent hypernuclear experiments [6]. The $g_{\sigma K}$ is related to the $K^{-}$optical potential depth $U_{K}$ $\left[=-\left(g_{\sigma K} \sigma+g_{\omega K} \omega_{0}\right)\right]$ in symmetric nuclear matter. We consider three cases: $U_{K}=-80 \mathrm{MeV}$ (weakly attractive), $-120 \mathrm{MeV}$ (moderately attractive), and $-180 \mathrm{MeV}$ (extremely attractive). See [4] for details of the parameters.

\section{Outline of multi-strangeness nuclei and $Y+K$ phase}

With regard to multi-strangeness nuclei (abbreviated to MSN), several $K^{-}$mesons are supposed to be put into a reference nucleus with the mass number $A$ and the number of protons $Z$. The number of initially input $K^{-}$mesons is denoted as $|S|$. Under the strangeness conservation, part of the strangeness $|S|$ initially carried by the $K^{-}$mesons is taken over by hyperons through $K^{-}+p \rightarrow \Lambda, K^{-}+n \rightarrow \Sigma^{-}$, $K^{-}+\Lambda \rightarrow \Xi^{-}$, and nonmesonic processes $\Lambda+\Lambda \rightarrow \Xi^{-}+p, \Lambda+\Sigma^{-} \rightarrow \Xi^{-}+n$. (We take into account only $\Lambda, \Sigma^{-}$, and $\Xi^{-}$for hyperons.) Then the ground state is expected to be composed of $K^{-}$ mesons with mixed hyperons in the nucleus, in chemically equilibrium with respect to the above strong reactions. Under a local density approximation for baryons, the thermodynamic potential $\Omega$ for the MSN with spherical symmetry is derived $[2,4]$. Together with the classical $K^{-}$field equation, one obtains the coupled equations of motion for the other mesons and the Poisson equation for $V_{\mathrm{Coul}}(r)\left(=-e A^{0}\right)$, with the constraints of baryon number $A$, electric charge $Z-|S|$, and strangeness $|S|$ conservations.

For the $Y+K$ phase realized in neutron stars, all the quantities are set to be uniform. In addition, the ultra-relativistic electrons participate and the Coulomb potential $V_{\text {Coul }}(r)$ is neglected. The ground state 


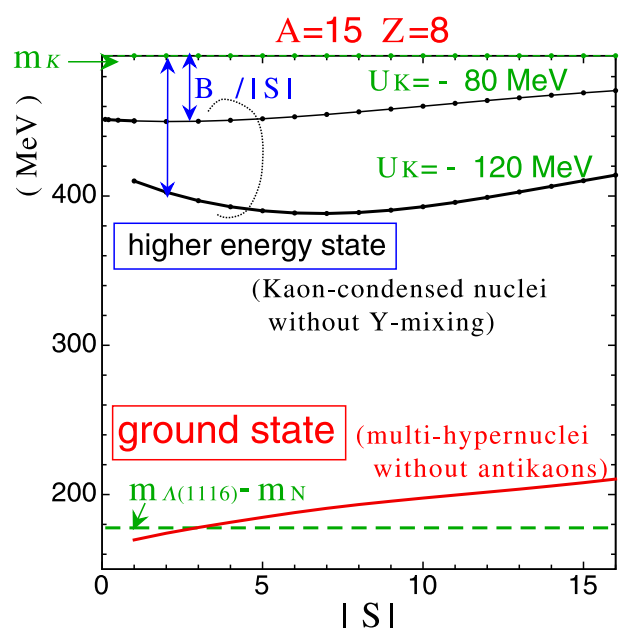

Figure 1. The energy per unit of strangeness $|S|$ for the MSN. The reference nucleus is taken to be ${ }_{8}^{15} \mathrm{O}$.

is obtained under chemical equilibrium conditions for weak processes: $n \rightleftharpoons p+K^{-}, n \rightleftharpoons p+e^{-}\left(\bar{v}_{e}\right)$, $p+e^{-} \rightleftharpoons \Lambda\left(v_{e}\right), n+e^{-} \rightleftharpoons \Sigma^{-}\left(v_{e}\right), \Lambda+e^{-} \rightleftharpoons \Xi^{-}\left(v_{e}\right)$, together with the charge neutrality and baryon number conservation.

\section{Numerical results}

\subsection{Muti-strangeness nuclei}

We summarize, in Fig. 1, the result of the energy per unit of strangeness $|S|, \Delta E /|S| \equiv[E(A, Z,|S|)-$ $E(A, Z, 0)] /|S|$, for the MSN as functions of $|S|$ in the case of a reference nucleus ${ }_{8}^{15} \mathrm{O}$.

In the ground state of the MSN, the whole strangeness is carried by hyperons with no bound $K^{-}$ mesons (multi-hypernuclei). The lowest energy of the input $K^{-}$mesons, $\omega_{K^{-}}$, is as much as $400 \mathrm{MeV}$, which is not much reduced from the free kaon mass (494 MeV), since $K^{-}$mesons do not have much attraction from the surrounding medium with central density $\rho_{c} \sim \rho_{0}$. Hence all the $K^{-}$mesons are absorbed by the nucleons and are taken over by the hyperons $(\Lambda)$ through $K^{-} N \rightarrow Y$. For $|S|>3$, $\Delta E /|S|$ exceeds the mass-difference between the $\Lambda(1116)$ and nucleon, so that the MSN decays through strong processes.

It is to be noted that the MKN with no hyperon-mixing [2] should be considered as the higher energy state. In the case of the MKN, the protons are attracted to the $K^{-}$mesons lying around the center due to the $I=0 \bar{K}-N$ interaction, so that both protons and $K^{-}$mesons become dense around the center of the MKN with $\rho_{c}=(3-4) \rho_{0}[2]$. As a result, the binding energy per unit of $|S|, B /|S|\left(\equiv m_{K}-\Delta E /|S|\right)$, amounts to several tens of $\mathrm{MeV}$ for $U_{K}=-80 \mathrm{MeV}$ and $\sim 100 \mathrm{MeV}$ for $U_{K}=-120 \mathrm{MeV}$.

\subsection{Kaon condensation in hyperonic matter $(Y+K$ phase $)$}

In Fig. 2a, the dependence of the lowest energy of $K^{-}$mesons $\omega_{K^{-}}$on baryon number density $\rho_{\mathrm{B}}$ is shown for $U_{K}=-80 \mathrm{MeV},-120 \mathrm{MeV}$, and $-180 \mathrm{MeV}$. The solid lines are obtained for hyperonic matter, while the dotted lines for nucleon matter. The dependence of the charge chemical potential $\mu\left(=\mu_{e}=\mu_{K^{-}}\right)$on $\rho_{\mathrm{B}}$ is also shown. The onset density of kaon condensation is obtained from the condition, $\omega_{K^{-}}=\mu$. (i) Once hyperons appear, the increase in the charge chemical potential $\mu$ is suppressed as $\rho_{\mathrm{B}}$ increases [1], while it steadily increases with $\rho_{\mathrm{B}}$ in nucleon matter. (ii) The decrease 

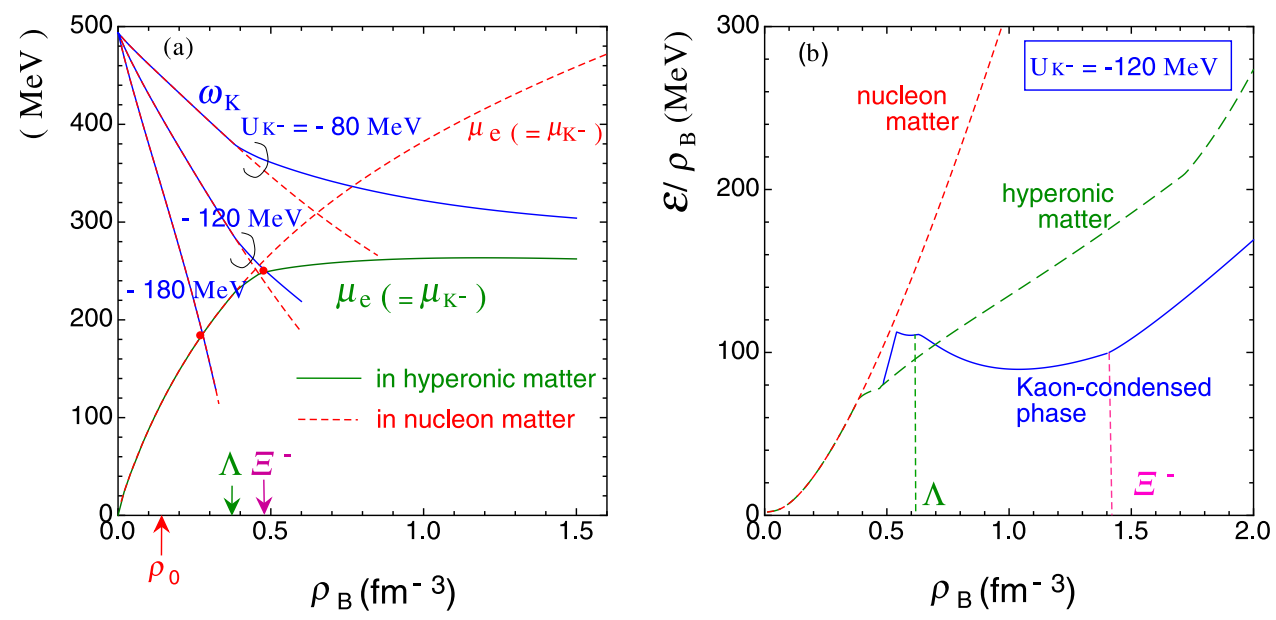

Figure 2. (a) The dependence of the lowest energy of $K^{-}$mesons $\omega_{K^{-}}$on baryon number density $\rho_{\mathrm{B}}$ for $U_{K}=-80 \mathrm{MeV},-120 \mathrm{MeV}$, and $-180 \mathrm{MeV}$. (b) The energy per baryon of the $Y+K$ phase (solid line) for $U_{K}=-120 \mathrm{MeV}$, The one of hyperonic matter without kaon condensation and the one in nucleon matter are also shown.

in $\omega_{K}$ with $\rho_{\mathrm{B}}$ gets moderate in the presence of hyperons, since the $K^{-}-Y$ vector interaction corresponding to $X_{0}$ does not contribute attractively to $\omega_{K}$. Owing to the results (i) and (ii), one can see from Fig. 2a that, in the case of $U_{K}=-80 \mathrm{MeV}$, kaons are not condensed in hyperonic matter over the relevant densities. For $U_{K}=-120 \mathrm{MeV}$, kaon condensation sets in at $\rho_{\mathrm{B}} \sim 3.3 \rho_{0}$. In case $U_{K}$ is very attractive $\left(U_{K}=-180 \mathrm{MeV}\right)$, kaon condensates appear before the onset of hyperons. In the case of hyperonic matter, the $K^{-}-B$ interaction sufficiently works to lower the $K^{-}$energy $\omega_{K^{-}}[=O(200 \mathrm{MeV})]$ near the onset density, which is in contrast with the case of the MSN.

In Fig. $2 \mathrm{~b}$, the energy per baryon for the $Y+K$ phase (solid line) is shown as function of $\rho_{\mathrm{B}}$ for $U_{K}=-120 \mathrm{MeV}$, together with the one in hyperonic matter without kaon condensation and the one in nucleon matter. Once kaon condensation occurs in hyperonic matter, it leads to significant softening of the EOS as a result of both the effect of attraction coming from the $s$-wave $K^{-}-B$ interaction and the effect of avoiding $N-N$ repulsion by mixing of hyperons.

\section{Concluding remarks}

It depends upon the magnitude of the $K^{-}-B$ attraction simulated by $U_{K}$ whether kaon condensation occurs in hyperonic matter. Recently neutron stars with mass as much as $2 M_{\odot}$ have been observed [7]. If the EOS with the $Y+K$ phase is to be consistent with these observations, primary effects of stiffening the EOS of the $Y+K$ phase at high densities should be taken into account. For example, many-body forces between baryons such as universal three-body repulsions between baryons may be indispensable [8]. It is also interesting to consider anti-symmetrization effect for baryons beyond the present Hartree approximation through introduction of tensor coupling of vector mesons [9].

The work is supported in part by the Grant-in-Aid for Scientific Research (No. 23540318) and by the funds provided by Chiba Institute of Technology. 


\section{References}

[1] T. Muto, Phys. Rev. C 77, 015810 (2008), and references cited therein

[2] T. Muto, T. Maruyama, T. Tatsumi, Phys. Rev. C 79, 035207 (2009)

[3] D. Gazda, E. Friedman, A. Gal, J. Mares, Phys. Rev. 76, 055204 (2007) Phys. Rev. 80, 035205 (2009)

[4] T. Muto, T. Maruyama, T. Tatsumi, JPS Conference Proceedings of The 12th Asia Pacific Physics Conference, (2014), to be published

[5] D.B. Kaplan, A.E. Nelson, Phys. Lett. B 175, 57 (1986)

[6] E. Botta, T. Bressani, G. Garbarino, Eur. Phys. J. A 48, 41 (2012)

[7] P.B. Demorest et al., Nature 467, 108 (2010); J. Antoniadis et al., Science 340, 1233232 (2013)

[8] S. Nishizaki, Y. Yamamoto, T. Takatsuka, Prog. Theor. Phys. 108, 703 (2002)

[9] T. Miyazu, T. Katayama, K. Saito, Phys. Lett. B 09, 242 (2012) 